\title{
Syndromic and sporadic pediatric optic pathway gliomas: review of clinical and histopathological differences and treatment implications
}

\author{
Mohammed F. ShamJi, M.D., M.Sc., ${ }^{1,2}$ AND Brien G. BenOIT, M.D., M.Sc., F.R.C.S.C. ${ }^{1}$ \\ ${ }^{1}$ Division of Neurosurgery, The Ottawa Hospital, Ottawa, Canada; and ${ }^{2}$ Department of Biomedical \\ Engineering, Duke University, Durham, North Carolina
}

\begin{abstract}
$\checkmark$ Optic pathway gliomas (OPGs) are the most common primary neoplasm of the optic pathway. These lesions usually present in childhood and can arise anywhere along the optic pathway; they occur more frequently in women; and they rarely undergo late progression. Management strategies after the initial diagnosis are controversial, compounded by the different behaviors exhibited by sporadic and syndromic tumors. Neurofibromatosis Type 1 (NF1), with aberrant oncogenic signaling and consequent predisposition to intracranial tumors, is the most common associated syndrome, with nearly $20 \%$ of NF1 patients developing OPGs. A comorbid NF1 diagnosis has implications for tumor location with greater predilection for optic nerve involvement, whereas chiasmal and postchiasmal lesions are more frequently seen in sporadic cases. Syndromic OPGs often exhibit more indolent behavior and lower rates of clinical progression, and the majority of these are diagnosed by routine neuroophthalmological screening. When treatment is indicated, however, the molecular abnormalities that constitute this syndrome can limit the available chemotherapy and radiotherapy options because clinicians fear secondary malignancy and cerebrovascular complications. Furthermore, radiotherapy early in life can impair an individual's intellectual development, endocrine function, and physical growth, thereby limiting the role of this modality in the treatment of this childhood lesion. Differential gene expression and histogenesis among sporadic and syndromic OPGs may account for the different tumor behaviors, but studies correlating specific genetic and proteomic changes with patient outcome are pending. Loss of heterozygosity at 10 and $17 \mathrm{q}$ are more common among patients with NF1, and Ki67 labeling intensity of 2-3\% and low p53 labeling intensity seem prognostic of aggressive tumor behavior. Recent advances in the development of a preclinical mouse model of NF1associated OPG will permit investigation into improved detection strategies and chemotherapeutic and radiotherapy treatment protocols. (DOI: 10.3171/FOC-07/11/E3)
\end{abstract}

KEY WORDS • glioma • neurofibromatosis • optic pathway • orbital tumor

$\mathrm{T}$ HE OPG is an intrinsic brain tumor that is the most common primary neoplasm of the optic pathway. These lesions typically manifest in early childhood, representing $1 \%$ of all brain tumors and up to $5 \%$ of brain tumors in children. Morbidity associated with OPGs can range from proptosis and cosmetic deformity to visual impairment, neurological and endocrine deficits, and death, with symptoms occurring in $\geq 20 \%$ of patients. ${ }^{60}$ The OPG can arise anywhere in the optic pathway, and several cases of retrogeniculate lesions have been reported. ${ }^{59}$ Younger patients typically present with slow-growing, WHO Grade I tumors that are commonly expansive and rarely metastasize, whereas adults are more likely to have higher-grade,

Abbreviations used in this paper: $\mathrm{NF} 1=$ neurofibromatosis Type 1; $\mathrm{MR}=$ magnetic resonance; $\mathrm{NIH}=$ National Institutes of Health; $\mathrm{OPG}=$ optic pathway glioma; $\mathrm{PA}=$ pilocytic astrocytoma; $\mathrm{VEP}=$ visual evoked potential; WHO = World Health Organization. malignant lesions that can directly invade adjacent structures, including the basal ganglia, hypothalamus, and internal capsule. Whereas pediatric lesions frequently do not progress, young patients with OPG have been shown to develop malignant degeneration, ${ }^{88}$ metastasis, ${ }^{64}$ and progression to glioblastoma multiforme, according to scattered reports. ${ }^{90}$ Syndromic and sporadic OPGs in the pediatric population may have different patient demographics, presenting symptoms, imaging features, and treatment outcomes that may mandate different clinical approaches. Furthermore, genetic profiling of these lesions suggests biological heterogeneity among the two groups.

\section{Syndromal Associations Associated With OPGs}

The most common syndrome associated with OPGs is NF1, also known as von Recklinghausen disease or peripheral NF. The incidence of OPG among this group has been estimated in neuroimaging studies to be between 15 and 20\%. ${ }^{9,51,52}$ The NIH consensus statement on NF in 1987 
M. F. Shamji and B. G. Benoit

established OPG as one of the seven diagnostic criteria defining the syndrome. ${ }^{2}$ This clinically heterogeneous neurocutaneous syndrome is the most common autosomal dominant disorder involving the nervous system, affecting 1 in 3000 individuals. The NF1 gene belongs to chromosome 17q11.2 and encodes a 2818 amino acid neurofibromin protein of 220 to $250 \mathrm{kD}$ molecular weight. ${ }^{13,65}$ Deactivating mutations, arising de novo in 30 to $50 \%$ of patients, are fully penetrant, leading to loss of function of the protein product. Neurofibromin normally serves as a negative growth regulator for astrocytes ${ }^{4}$ by acting as a guanosine triphosphate GTPase-activating protein, ${ }^{91}$ accelerating the conversion of intracellular active Ras-GTP to inactive Ras-GDP and hence attenuating downstream Rasmediated signaling. Indeed, Lau and coworkers ${ }^{49}$ have reported the case of an NF1-associated pilocytic astrocytoma in which loss of neurofibromin expression correlated with high levels of Ras-GTP and its downstream mediators. Similar elevated levels were demonstrated by Sherman and coworkers ${ }^{77}$ in an NF1-associated neurofibroma. Other ophthalmological manifestations of NF1 include Lisch nodules of the iris, neurofibromas of the eyelids and orbit, sphenoid dysplasia, retinal tumors, and multifocal choroidal nevi. ${ }^{23,40,73}$ Table 1 provides a summary of the NIH diagnostic criteria for NF1.

Several challenges have occurred during the development of a preclinical animal model of optic pathway tumorigenesis. Mouse models of NF1 have been well reviewed. ${ }^{35}$ Animals that are deficient in the neurofibromin protein $(N F 1-/-)$ experience embryonic lethality and hence cannot serve as experimental subjects. ${ }^{11,41}$ Whereas heterozygous animals $(\mathrm{NFI+l}-)$ are tumor prone, they are observed to demonstrate astrocyte hyperplasia ${ }^{37}$ but have not been observed to develop gliomas. ${ }^{41}$ A novel transgenic mouse model of NF1 that develops optic nerve and chiasmal gliomas has permitted Bajenaru and coworkers ${ }^{5,6}$ to examine the natural history of these lesions. These animals are heterozygous $(N F 1+/-)$ and have conditional knockout of NF1 expression in astrocytes..$^{92}$ Histological studies have demonstrated the onset of astrocyte hyperplasia, neovascularization, and microglial infiltration as early as 3 weeks, with overt neoplastic changes present at 2 months. Immunohistochemical testing shows high expression of proteins related to astroglial progenitors, including nestin and brain lipid binding protein. Diffusion tensor MR imaging at 2 months demonstrated development of gliomas in only NF1 heterozygous animals with astrocyte NF1 knockout but required nearly 3 hours of data acquisition. Manganeseenhanced MR imaging leads to tumor enhancement by axonal transport of the contrast agent and also abbreviates the time needed for reliable OPG detection to 10 to 15 minutes of imaging time. ${ }^{8}$ The value of this animal model will be in the ongoing elucidation of molecular events involved in the development of this tumor among patients with NF1, as well as the preclinical sensitivity of these lesions to chemoand radiotherapy.

There are rare case reports coupling OPGs with other syndromal conditions. Sheta and coworkers ${ }^{78}$ have described a 14-year-old girl with a history of colonic polyposis who went on to develop unilateral proptosis eventually attributed to a WHO Grade I pilocytic astrocytoma. Treatment involved lateral orbitotomy and excisional biopsy. The authors proposed the association of Turcot syndrome
TABLE 1

Diagnostic criteria for $\mathrm{NF} 1$

\begin{tabular}{ll}
\hline \multicolumn{1}{c}{ Criteria } & \multicolumn{1}{c}{ Specifics } \\
\hline cafe-au-lait macules & $\geq 6$ \\
neurofibromas & 1 plexiform neurofibroma \\
axiallary or inguinal freckling & present \\
optic pathway glioma & present \\
Lisch nodules (iris hamartomas) & $\geq 2$ \\
bone lesion & $\begin{array}{l}\text { sphenoid wing dysplasia, thinning of } \\
\text { log-bone cortex } \\
\text { first degree }\end{array}$ \\
\hline
\end{tabular}

of familial intestinal polyposis and primary neuroepithelial tumors to be manifest in this case as an OPG and further advocated that both ophthalmologists and gastroenterologists consider this association once 1 of the criteria is met. Erbay and coworkers ${ }^{27}$ have reported on a rapidly developing OPG in a patient with hybrid phacomatosis, combined disease of both NF1 and tuberous sclerosis. Coexistent phacomatoses that can have additive or synergistic genetic effect may explain the rapid evolution of an OPG, absent on MR imaging at 3 months, but present at age 8 months as clinical proptosis with MR imaging demonstration of an enlarged right optic nerve.

\section{Clinical Features}

\section{Demographics and Presentation}

It is commonly held that NF1-associated OPGs are characterized by a more indolent course, with patients less likely to experience neurological progression. This may result in part because of different status of syndromic and sporadic OPG patients at the time of medical evaluation. Most series in which authors have compared the natural history of these lesions acquire patients sporadically after the individuals present with visual symptoms, and NF1 patients present in an asymptomatic state after routine neuroophthalmological screening. ${ }^{3,15,20,33,52,61}$ Nevertheless, common demographic features in many of these studies include higher numbers of female than male OPG diagnoses and earlier age of presentation for NF1 patients. ${ }^{3,20,44,79}$ The incidence of specific presenting symptoms in symptomatic OPG patients is also consistently reported. The most common clinical feature is decreased visual acuity, $3,15,20,33,46$, 55,61 with greater incidence among patients with sporadic OPGs. ${ }^{20,33,55}$ Abnormalities in the ophthalmological examination occur with variably reported incidence but most commonly manifest as optic disc atrophy with a similar frequency regardless of NF1 status. ${ }^{55,61}$ Observed exophthalmos is related to the retrobulbar location of the optic nerve glioma and has been reported to be more common in NF1 patients $^{22,33,61}$ although the opposite trend has also been reported. ${ }^{20}$ Patients with sporadic lesions are more likely to present with features of raised intracranial pressure. ${ }^{3,20,55}$

Listernick and coworkers ${ }^{52}$ have studied the natural history of these lesions, specifically among NF1 patients, and have reported that only half of asymptomatic children will ever develop symptoms or signs related to the disease. Clinical progression in those who presented with symptoms was only observed in $33 \%$ of patients, nearly all of 
them occurring before the age of 6 years. This pattern makes straightforward comparison of sporadic and syndromic OPG patients very difficult in cohort studies. King and coworkers ${ }^{44}$ evaluated patients with NF1 who were brought to OPG care and compared their symptomatic and asymptomatic patients. The mean age at presentation was 4.8 years and 5.4 years for the 51 symptomatic and the 39 asymptomatic patients, respectively. Common symptoms included proptosis $(26 \%)$ and headache $(8 \%)$; precocious puberty affected $20 \%$ of individuals; and in $54 \%$ of symptomatic patients ophthalmological examinations yielded abnormal results (most commonly decreased visual acuity). There were no significant differences between NF1 patients who presented with symptoms (51) or without symptoms (39) with regard to age at NF1 and OPG diagnoses, location of the OPG, or associated NF1 features. A similar comparison of 32 symptomatic and 22 asymptomatic NF1associated OPG patients was reported by Thiagalingam and coworkers. ${ }^{83}$ The mean ages of presentation of 4.8 and 5.8 years for the symptomatic and asymptomatic groups, respectively, were not significantly different. They also observed high rates of vision loss (23 of 32) and proptosis (10 of 32), with identifiable optic disc atrophy in 25 of their symptomatic patients. In both of the aforementioned studies, patients with NF1 who presented with symptoms were more likely to require initial treatment. ${ }^{44,83}$ A study comparing demographics, presentation, and outcomes of the symptomatic NF1 subgroup with a sporadic control cohort is needed.

\section{Imaging and Lesion Location}

Optic nerve gliomas usually appear as well-demarcated lesions on computed tomography scanning. Calcification is rare, and this can differentiate the intraaxial OPGs from optic nerve meningiomas that frequently calcify. The rare case of a calcified glioma is more likely to occur in the chiasm than the nerve..$^{70}$ Magnetic resonance imaging reveals lesions that are isointense or hypointense to cerebral white matter on T1-weighted sequences, with areas of necrosis or mucinous degeneration that are markedly hypointense. The T2-weighted sequences reveal hyperintense gliomas. Contrast enhancement with Gd can be homogeneous or heterogeneous. Contrast-enhanced T1- or T2-weighted images are best for visualizing posterior extension into the optic tracts. ${ }^{70}$

Syndromic and sporadic OPGs have discrete anatomical patterns and lesion characteristics on MR imaging. Kornreich and coworkers ${ }^{45}$ have used MR imaging to examine 91 patients with OPG with the goal of comparing imaging findings and determining prognostic features. It is important to note that all 44 patients with sporadic tumors were referred because of symptoms, whereas only $32 \%$ of the 47 NF1 patients were symptomatic and the rest were discovered during routine screening procedures. Lesions in patients with the sporadic form of the disorder predominantly had a chiasmatic location (92\%) with optic nerve involvement in only $32 \%$, cystic morphology in $66 \%$, and deformed normal shape of the optic pathways in $73 \%$. Conversely, the NF1 patients had lesions most commonly located at the optic nerve (66\%) with $62 \%$ involving the chiasm, $9 \%$ rarely having cystic components, and $91 \%$ frequently having no effect on the shape of the optic pathway.
The cystic morphology may be clinically significant, accounting for more than $50 \%$ of the tumor size in patients with cystic OPGs. Patients with the sporadic form commonly harbored tumors that extended beyond the optic pathways (68\%), and hydrocephalus was a common finding $(47 \%)$. Only one NF1 patient $(2 \%)$ had a tumor with extraoptic extension, and none of these patients had hydrocephalus. There were no identifiable imaging features of prognostic significance. Chateil and coworkers ${ }^{15}$ similarly reviewed 27 patients with OPG, 14 with a diagnosis of NF1 and 13 with a diagnosis of sporadic lesions. Again, all patients with the sporadic form were symptomatic at presentation, whereas only $29 \%$ of patients with NF1 had complaints attributable to their tumor. All patients with sporadic lesions had tumors involving the chiasm, and 54\% had a cystic component. These tumors were round, delineated masses that homogeneously enhanced after administration of contrast medium. Seventy-one percent of NF1 patients had tumors involving the optic nerve, and no cystic changes were observed. Tumor proliferation leads to nerve elongation enlarging diffusely into a tubular widening that eventually becomes tortuous. The larger tumor size in the sporadic group is probably the reason for the symptomatic status of these patients compared with the syndromic group. In both series, bilateral OPGs were observed with higher frequency among patients with NF1..$^{15,45}$ It is interesting to note that Thiagalingam and coworkers ${ }^{83}$ differentiated NF1 patients by their symptomatology at presentation and found that $75 \%$ of symptomatic patients had some chiasmal involvement, implicating this as an important site of symptom onset. Conversely, King and cowork$\mathrm{ers}^{44}$ reported no difference in OPG location between their symptomatic and asymptomatic patients with NF1.

Tumor location is strongly related to ophthalmological morbidity. ${ }^{25}$ Although postchiasmatic lesions are rare, patients in this group suffer from the highest incidence $(62 \%)$ of visual acuity loss. Isolated optic nerve and isolated chiasmal lesions exhibit only 25 and $33 \%$ prevalence of this deficit, respectively. ${ }^{7}$ There is no consensus in the literature regarding the predictors of visual deficit progression after the initial diagnosis in NF1 patients. ${ }^{7,52}$

\section{Treatment Options and Outcomes}

Surgical treatment of OPGs is generally reserved for cases in which the abnormal tissue generates symptoms attributable to its mass. Resection of the optic nerve or chiasmal tumors is associated with significant neuroophthalmological morbidity and therefore is undertaken only in cases in which the lesion size introduces its own morbidity. Surgical indications include onset of obstructive hydrocephalus, disfiguring proptosis and associated corneal exposure, and severe and irreversible vision loss in the rare tumor that exhibits aggressive behavior. ${ }^{3,56,58}$ One of the largest surgical series of OPG patients was reported by Ahn and coworkers, ${ }^{1}$ who described outcomes in 33 patients at a mean follow-up duration of 52 months. Most common complaints included visual disturbances $(97 \%)$ and headache with vomiting (57\%), and the goal of surgery was to debulk the tumor mass and to improve mass effect-related symptoms. Ipsilateral vision preservation was achieved in $25 \%$, with no differences between patients who underwent radical or partial removal. Preoperative hydrocephalus in 
M. F. Shamji and B. G. Benoit

20 patients was resolved in $70 \%$, with six patients requiring a shunt placement. The authors proposed surgery as primary treatment in cases of optic nerve lesions in which the patient had poor vision or in cases of chiasmal lesions in which the patient had useless vision on the poor-vision side, ${ }^{2}$ although this recommendation is not universal. ${ }^{56}$ Table 2 provides a summary of the current evidencebased recommendations for the treatment of children with NF1 and OPG adapted from Listernick and coworkers. ${ }^{56}$ Differences in the management of sporadic OPGs will be discussed when addressing each modality later.

Given the propensity of these lesions to exhibit indolent behavior, the primary management of nonprogressive, nonsurgical lesions is close observation. Frequent ophthalmological follow-up of these pediatric patients should be performed at 3-month intervals for the 1st year after diagnosis followed by increasing intervals after that. ${ }^{56}$ Magnetic resonance imaging follow-up is equally important, although guidelines about its frequency have not yet been established. Listernick and colleagues ${ }^{57}$ have described 8 patients with NF1 who had either late-onset or late-progressive OPGs and articulated the need for continued monitoring of known OPGs into adulthood. Asymptomatic NF1 patients with OPG generally come to medical attention after routine neuroophthalmological screening, and in these patients low rates of disease progression are consistently found. Thiagalingam and coworkers ${ }^{83}$ have reported on 2 of 22 asymptomatic patients with progression of signs at 2 and 6 years after diagnosis, whereas in 26 of 32 symptomatic patients disease progressed and specific treatment was required for 15 . Similarly, King and coworkers ${ }^{44}$ have described clinical progression requiring treatment in only 1 of 39 asymptomatic patients. Eighteen of 51 initially symptomatic patients required specific treatment that included surgery or chemotherapy. As a consequence of the heterogeneity of reporting centers and patient clinical status at presentation, the reported rates of disease progression have been quite variable. In a series of 106 patients, the age at initiation of specific treatment required for clinical or imaging progression was significantly lower in patients with sporadic lesions than in those with NF1. ${ }^{33}$ The event triggering treatment was most commonly visual loss in both groups, but a greater number of sporadic cases required treatment for raised intracranial pressure. In patients who initially underwent conservatively follow-up for mild symptoms, 12 of 20 patients with NF1 and all of the 12 with sporadic tumors suffered disease progression at follow-up. Smaller studies support the surveillance strategy, with a significant fraction of both symptomatic and a majority of asymptomatic NF1 patients exhibiting stable disease over years of follow-up. . $^{12,20,22,52,55,83}$

In cases of tumor progression when surgery is not primarily indicated, the choice of therapy differs depending on if the case involves sporadic or syndromic OPGs. The therapeutic approaches differ because of the molecular aberrations associated with the NF1 comorbidity. Radiotherapy has historically been undertaken for control against tumor growth and has occasionally effected visual symptom resolution. In many series, radiotherapy has been used as primary or postsurgical adjuvant therapy for both syndromic and sporadic OPGs in patients older than 5 years, $3,20-22,33,61$, $67,79,83$ with some authors advocating specific practice guidelines independent of NF1 status. ${ }^{50}$ Indeed, the NF1 Optic
TABLE 2

Follow-up and treatment of children with NF1 and $O P G^{*}$

\begin{tabular}{cl}
\hline \hline $\begin{array}{c}\text { Treatment } \\
\text { Modality }\end{array}$ & \multicolumn{1}{c}{ Indications } \\
\hline observation & $\begin{array}{c}\text { 1) for nonprogressive disease, FU should be in centers w/ } \\
\text { pediatric ophthalmologists or neuroophthalmologists } \\
\text { w/ experience of NF1 }\end{array}$ \\
& $\begin{array}{l}\text { 2) ophthalmological exams every } 3 \text { mos for } 1 \mathrm{yr} \mathrm{w} / \mathrm{in}- \\
\text { creasing intervals thereafter }\end{array}$ \\
& $\begin{array}{l}\text { 3) MR image at similar intervals depending on institu- } \\
\text { tional preference }\end{array}$ \\
chemotherapy & 1st line therapy for progressive disease \\
radiotheraphy & $\begin{array}{l}\text { not recommended because of risk of secondary malig- } \\
\text { nancies \& radiation-induced vasculopathy }\end{array}$ \\
surgery & 1) removal of intraorbital optic nerve indicated for cos- \\
& mesis or to treat corneal exposure
\end{tabular}

$\mathrm{FU}=$ follow-up.

Pathway Glioma Task Force paper published in 1997 also advocated the use of radiotherapy in patients with NF1associated isolated progressive intraorbital OPGs when residual useful vision was present. ${ }^{58}$ Long-term follow-up data provided by Cappelli and coworkers ${ }^{12}$ of 69 patients treated exclusively with surgery and/or radiotherapy revealed a 10 -year overall survival rate in $83 \%$, with no differences between syndromic and sporadic lesion if the start date was taken as the time of radiotherapy. However, complications were more prevalent in patients with NF1 and included cerebrovascular occlusion and poorer intellectual function. Other authors have reported high relative risk of second primary brain tumors (relative risk $=3.04$ ) (6) $^{76}$ and the occurrence of cerebral occlusive vasculopathy ${ }^{32,85}$ among NF1 patients in whom OPGs were treated with radiotherapy. Radiotherapy is no longer recommended for the treatment of these lesions in patients with NF1 $1{ }^{56}$ Recent investigation into stereotactic radiosurgery (52.2-Gy dose) involving a 97-month follow-up revealed a $90 \%$ 5-year survival rate with no endocrine abnormalities and no second primary brain tumors. ${ }^{18}$ This series of 15 patients includes only 3 NF1-associated lesions, suggesting that further investigation into this treatment modality is required.

Chemotherapy has typically been reserved for children $<$ age 5 years requiring treatment. This is because young patients are at high risk of cognitive, endocrine, and developmental defects after radiation is delivered to large volumes of the brain..$^{50}$ The most common regimen is carboplatin/ vincristine combination therapy, which has been shown to be effective in controlling disease progression. Packer and coworkers ${ }^{66}$ reported that $56 \%$ of their 31 patients responded to treatment, with no significant difference in 2-year progression-free survival between NF1-associated (79\%) and sporadic (75\%) nonresectable gliomas. More recently, Gnekow and coworkers ${ }^{30}$ evaluated this regimen to delay the need for radiotherapy in 123 patients with chiasmatic and/or hypothalamic gliomas and a median age of 3.6 years. They also observed high rates of remission and disease stability, with only 44 patients having progressive tumors after a median of 22.5 months. Sixteen of these patients subsequently underwent radiotherapy, but the initial chemotherapy achieved the objective of delaying the need for more toxic radiotherapy in these very young pa- 
tients. Carboplatin monotherapy has had limited success, with Mahoney and coworkers ${ }^{63}$ reporting no more than $30 \%$ response in 51 children age 5 years or younger. They observed no differences between patients with NF1 and sporadic lesions. Listernick and coworkers ${ }^{54}$ also described 9 patients with NF1 who underwent carboplatin monotherapy, among whom 2 experienced improved vision and 4 exhibited imaging-documented improvement. Other antineoplastic drugs such as temozolomide alone ${ }^{34,47}$ or lomustine $^{42}$ are active against these tumors, but such alkylating agents have induced secondary malignancy in the NF1 mouse model and thus have contraindications. ${ }^{62}$ The use of these agents in patients with sporadic OPGs may be an effective alternative in individuals who cannot tolerate carboplatin and vincristine or whose tumors are resistant to them. Given the new data surrounding the unacceptable risks of radiotherapy in patients with NF1, chemotherapy is now regarded as the primary treatment modality in those with progressive, nonsurgical NF1-associated OPGs. ${ }^{56}$ Nevertheless, such treatment carries its own risk of neuropsychological complications. Lacaze and coworkers ${ }^{48}$ have described maintenance of intellect in patients with sporadic OPGs treated with chemotherapy, but they observed decreased intelligence quotient scores and greater memory-related deficits among patients with sporadic lesions treated with radiotherapy, as well as in NF1 patients treated with chemotherapy.

Relapse-free and overall survival rates among patients with OPGs are variably reported in small case series. The authors of studies comparing sporadic and syndromic OPGs have reported similar and favorable 5- and 10-year survival, ${ }^{22,79}$ although specific rates are variable and based on the treatment center and management algorithms. Tow and coworkers ${ }^{84}$ have reported results after a minimum of a 10-year follow-up period (mean 14.9 years), and they observed $100 \%$ survival among 16 patients with isolated optic nerve lesions (50\% NF1), 94\% survival among 16 with chiasmal lesions (63\% NF1), and 93\% survival among 15 with chiasmal lesions with hypothalamic involvement $(27 \%$ NF1), but they did not differentiate between NF1 and sporadic lesions. Prior to the widespread availability of chemotherapy, Cappelli et al. ${ }^{12}$ reported that the 10-year survival in 69 patients (1980-1992) treated with radiotherapy and surgery, when indicated, was only slightly lower at $83 \%$ overall and that $65 \%$ were disease free. Although the authors concluded that radiotherapy provides therapeutic value in imaging treatment response and stabilization of visual symptoms, the development of chemotherapy and the known complications of brain irradiation early in life lead them not to recommend its use early in contemporary treatment algorithms. Chan and coworkers ${ }^{14}$ developed a multivariate model to evaluate specific prognostic factors in 69 children with OPGs. They concluded that both increasing age at presentation and NF1-positive status (present in 26 patients) were associated with lower likelihood of disease relapse. Radiotherapy or chemotherapy was also associated with such protection, even compared with the results in patients in whom primary treatment at diagnosis was not indicated. Sex and tumor location did not alter disease-free survival, nor did surgical intervention performed as first-line treatment in 13 patients.

Central precocious puberty is the most common endocrinopathy associated with NF1, with a reported prevalence of $3 \%$ compared with $0.06 \%$ in the general pediatric population. ${ }^{38}$ Similarly, growth hormone deficiency is reported to occur in $2.5 \%$ of NF1 patients, compared with $0.03 \%$ of the general pediatric population. These findings were initially attributed to local tumor mass effect compromising hypothalamic and pituitary function, as Habiby and coworkers $^{38}$ described in 7 of 18 patients with chiasmal glioma who developed central precocious puberty. Furthermore, they observed no cases of central precocious puberty in the absence of such a lesion. More recently, Cnossen and coworkers ${ }^{17}$ described such disturbances occurring in association with 2 of 9 chiasmal gliomas, but in only 1 of 3 patients with central precocious puberty and 1 of 3 with growth hormone deficiency. Causal mechanisms are unclear but may involve slow-growing lesions undetectable on neuroimaging or alternate manifestations of the NF1 molecular abnormalities leading to transcriptional dysregulation.

\section{Histopathology and Genetic Differences}

The WHO classifies OPGs as Grade I astrocytic neoplasms. These lesions demonstrate glial fibrillary acidic protein immunopositivity and are typically hypocellular, have rare mitoses, and have mildly hyperchromatic nuclei. Eosinophilic Rosenthal fibers may also be present. Nevertheless, the histological nature of OPGs is heterogeneous both between and within tumors, and Verhoeff ${ }^{87}$ was the first to classify 3 distinct glioma patterns. The coarsely reticulated, finely reticulated, and coarsely fibrillated subtypes are now recognized to be variants of PAs. ${ }^{19,25}$ The typical histological pattern is coarsely reticulated with biphasic morphology of bipolar astrocytes that are either tightly compacted around blood vessels or loosely organized around microcystic regions. There may be associated proteinaceous droplets, Rosenthal fibers, and eosinophilic granular bodies. Less common is the finely reticulated pattern characterized by small ovoid nuclei within a delicate syncytium of fibrils. This can be confused with a more aggressive WHO Grade II diffuse astrocytoma, and typical differentiation based on MR imaging is often difficult for lesions in this location. The coarsely fibrillar type is characterized by coarse neuroglial fibrils and spindle neuroglia arranged in definite bundles and is more typical of the adult variant of PAs. ${ }^{74}$ To address the issue of differentiating more indolent PAs from the more aggressive diffuse astrocytomas, Cummings and coworkers ${ }^{19}$ sought to identify biomarkers suggestive of an aggressive OPG phenotype in 22 patients in 5 of whom comorbid NF1 was present. They identify characteristics of hypothalamic or chiasmal location, Ki67 labeling intensity of 2-3\%, and p53 labeling intensity of less than $1 \%$ as positive prognostic indicators of disease progression. They observed that NF1 positivity did not manifest as protective, although the number of patients in this series was small. Furthermore, it was not made clear as to how these features should influence patient management beyond implying the need for more rigorous follow-up.

The natural history of OPG is reported consistently in the literature as having a favorable outcome in patients with NF1. However, many studies suffer from the complicating feature that patients with sporadic OPGs commonly seek medical attention because of ophthalmological symptoms, 
whereas those the NF1 more commonly present asymptomatically after routine neuroophthalmological screening and appropriate neuroimaging. A desirable statistical comparison, hitherto unreported in the literature, is to consider all OPG patients who are symptomatic with ophthalmological complaints. Indeed, King and coworkers ${ }^{44}$ have reported that patients with NF1-associated OPGs who are symptomatic at presentation are significantly more likely, with an odds ratio of 14.8, to require intervention. Even this type of study may suffer from the bias that physicians following NF1 patients may be more sensitive to the importance of quickly following up complaints of headache or visual disturbance because of common associations with OPGs and other astroglial tumors.

The histogenesis of PAs remains uncertain, although evidence suggests that they may arise from a different progenitor cell than diffuse astrocytomas. Pilocytic astrocytomas have been reported to express molecular markers typical of oligodendrocytes or multipotent $\mathrm{O} 2 \mathrm{~A}$ precursors that can then differentiate into astrocytes or oligodendrocytes. Of particular note is the expressed PEN5 epitope ${ }^{28}$ that is not expressed in high-grade astrocytomas. Conversely, Gutmann and coworkers ${ }^{36}$ used oligonucleotide array analysis to demonstrate that genes typically altered in fibrillary astrocytomas, such as p53, retinoblastoma, PTEN, p19, p16, $\mathrm{dmd} 2$, and cyclin-dependent kinase 4, were not affected in either sporadic or NF1 PAs. An intriguing result from their study was the identification of the $E F-1 \alpha 2$ gene that was increased in all sporadic PAs compared with the NF1-associated lesions. The EF1 $\alpha$ protein has been implicated in oncogenesis of rat mammary adenocarcinoma, ${ }^{26,71}$ although its significance for intrinsic central nervous system tumors remains uncertain. Mutation of the tumor-suppressing PTEN gene on chromosome 10 is rare among PAs, with only 1 case of 30 found having been found to be positive for such change. ${ }^{24}$ Tada and coworkers ${ }^{81}$ evaluated chromosomal changes in 12 PAs and demonstrated loss of heterozygosity of 17q in 1 of 9 sporadic PAs and 3 of 3 NF1associated PAs. Furthermore, loss of heterozygosity of chromosome 10 occurred in none of sporadic cases but in 2 of 3 NF1-associated lesions. These data support the claim that there are molecular differences in tumor oncogenesis for lesions in patients with NF1.

\section{Spontaneous Regression}

Spontaneous regression of OPGs has been anecdotally reported in the literature, although the incidence and clinical impact of such an event remain uncertain. Postoperative regression of residual lesions has been documented on computed tomography scanning, ${ }^{39,82,86}$ and MR imaging has demonstrated similar involution in both asymptomatic ${ }^{31,68}$ and symptomatic ${ }^{75}$ lesions. Histological evidence of such regression in biopsy-proven glioma as well as incompletely resected gliomas has been reported by Borit and Richardson. ${ }^{10}$ More recently, Parsa and coworkers ${ }^{69}$ reported a series of 13 patients acquired by surveys at the North American Neuro-Ophthalmology Society and requests at the NANOSNET listserv, all of whom exhibited imaging evidence of tumor shrinkage. Nine of these patients had sporadic lesions and three of these had a history of specific treatment including 1 who underwent radiotherapy 19 years previously, 1 who underwent chemotherapy 5 years previously, and 1 who underwent subtotal tumor resection 1 year previously. Visual field perception was reported to worsen in 2 of these patients, and tested acuity worsened in 1. Piccirilli and coworkers ${ }^{72}$ summarized the data culled from 16 cases from the English-language literature and obtained in 3 of their own patients, but the true incidence of this event is uncertain. The molecular mechanisms of this regression remains unknown and have been proposed to include endocrine-associated decreases in vascular tumor engorgement, immunogenically mediated tumor necrosis ${ }^{43}$ and imbalance between pro-apoptotic signals and lowgrade proliferative behavior. ${ }^{69}$ Surgery, including needle biopsy or partial resection, may unmask a previously immune-protected antigen, allowing the host's immune system to mount a cytotoxic reaction - and the aging process itself is associated with increasing immunocompetence in the child. ${ }^{43}$ Other authors believe that tumor proliferation may be outpaced by terminal differentiation and apoptosis and that surgical debulking in subtotal resection creates sufficient space to permit the regression process to occur. ${ }^{43,69,82}$ Although these case reports are rare, the low incidence of progression to symptomatic disease after childhood suggests the role of evolving immunocompetence in disease stabilization and underscores the need for close clinical and imaging observation in the management of asymptomatic lesions.

\section{Screening Strategies}

Screening of asymptomatic children known to have the NF1 phenotype for OPGs has been the subject of investigation. In the NF1 Optic Pathway Glioma Task Force study published in 1997, investigators have recommended annual ophthalmic examinations by a pediatric ophthalmologist or a neuroophthalmologist for all asymptomatic patients $<$ 6 years of age. ${ }^{58}$ The appropriate parameters of such examination has not been defined, but various methods used in clinical series include visual acuity, visual fields, and VEPs ${ }^{56}$ Visual acuity testing has high test-retest reliability, even in young children. ${ }^{16,29}$ It is the most reliable and clinically relevant measure of compromised visual function in OPGs. Infant testing involves preferential looking tests; preliterate children can be evaluated by figure or opotype ("HOTV") matching and older children by Snellen charts. Visual field abnormalities in children with OPGs are rarely seen without concomitant loss of acuity. ${ }^{53}$ The duration of these studies, and the attendant monotony, have been reported to lead to high numbers of false-positive and falsenegative results in young children..$^{80}$ These tests also have high test-retest variability, and consequently the clinical value of changes during serial testing remains questionable. Visual evoked potentials are a sensitive method of measuring the electrophysiological integrity of the optic pathway. The VEPs were found to be $86 \%$ sensitive - they increased to $93 \%$ when serial testing applied-for the detection of OPGs in 297 patients with NF1.89 The sensitivity of the testing, and the specificity of $75 \%$, leads to identification of abnormalities in many asymptomatic patients, as well as many false-positive OPG diagnoses. The evidence for VEPs as a screening tool is equivocal, but VEP monitoring may be of value when ophthalmic examinations are difficult. Current recommendations suggest that all patients with unexplained abnormal findings during ophthalmic 
screening undergo contrast-enhanced MR imaging of the head and orbits.

Blazo and coworkers ${ }^{9}$ have prospectively compared the impact of screening neuroimaging with screening by ophthalmic examination alone on visual outcomes. A previously unknown OPG was identified in $20 \%$ of 54 children who underwent imaging at a mean age of 2.8 years; and only 2 of the patients presented with visual abnormalities, 1 with strabismus and 1 with optic nerve atrophy. The mean age of patients was 3.6 years in a historical cohort of 13 children at Blazo and coworkers' center diagnosed with OPG outside of the neuroimaging screening study; 6 of them presented with abnormal ophthalmic examination results, including 4 patients with proptosis and visual impairment in the affected eye. To establish evidence-based guidelines for detecting OPGs in children with NF1, we need a specific prospective comparison of a neuroimaging screening protocol and the guidelines of the NF1 Task Force.

\section{Management of NF1-Associated OPGs}

Screening of NF1 patients $<6$ years of age by annual ophthalmic examinations should include visual acuity testing, ${ }^{56,58}$ although the evidence for including visual fields and VEPs remains uncertain. Neuroimaging screening may be more sensitive to detect the presence of OPG lesions, ${ }^{9}$ but there is no prospective evidence that this earlier detection affects neuroophthalmological or survival outcomes. Children who have abnormal ophthalmic examinations should undergo contrast-enhanced MR imaging to evaluate any intracranial lesions.

Treatment strategies for NF1-positive children with identified OPGs are summarized in Table 2. Briefly, patients with static disease should be followed up into adulthood, undergoing sequential ophthalmological examinations to detect worsening visual symptoms and signs, as well as periodic MR imaging to detect lesion growth. ${ }^{56-58}$ Although radiotherapy has been reported to improve visual function, ${ }^{12}$ the associations with impaired intellectual and physical development, endocrine dysfunction, and the risks of secondary malignancy and cerebral occlusive vasculopathy have led to its removal from the treatment algorithm for NF1-associated OPGs. An initial regimen of carboplatin and vincristine chemotherapy can be used as first-line treatment in cases of nonsurgical NF1-associated $\mathrm{OPGs}^{66}$ with the primarily goal of controlling disease and the secondary goal of delaying the need for radiotherapy. Other regimens continue to be investigated, but choices of chemotherapeutic agents are limited by the molecular abnormalities of NF1. Surgery should be offered to patients who have developed tumor mass effects such as obstructive hydrocephalus, for aggressively growing tumors in patients with severe vision loss, and for disfiguring proptosis with corneal exposure. ${ }^{56,58}$ The provision of surgery for histological analysis is seldom necessary, and surgical debulking rarely restores vision.

\section{Conclusions}

Optic pathway gliomas represent a clinically heterogeneous group of lesions that are most prevalent in the pediatric population. This tumor is one diagnostic criterion for NF1, and a significant number of patients with molecular abnormalities of neurofibromin signaling develop OPGs as well as other intracranial gliomas. The location of tumors, along the optic pathway, varies significantly between sporadic and syndromal OPG groups, and most studies have shown that the NF1 diagnosis is associated with differences in tumor location, manifesting more indolent behavior and lower likelihood of visual symptoms and clinical progression. In most of these studies the authors have compared symptomatic sporadic OPGs with both symptomatic and asymptomatic NF1-associated OPGs detected by neuroophthalmological screening. This prevents a rigorous comparison between the two groups, especially in the context of management strategies that continue to evolve divergently for the treatment of sporadic and syndromic lesions. Recent advances in a preclinical mouse model of NF1-associated OPG will permit investigation into improved detection strategies and chemo- and radiotherapy protocols.

\section{References}

1. Ahn Y, Cho BK, Kim SK, Chung YN, Lee CS, Kim IH, et al: Optic pathway glioma: outcome and prognostic factors in a surgical series. Childs Nerv Syst 22:1136-1142, 2006

2. Anonymous: National Institutes of Health Consensus Development Conference Statement. Neurofibromatosis 6:1-7, 1987

3. Astrup J: Natural history and clinical management of optic pathway glioma. Br J Neurosurg 17:327-335, 2003

4. Bajenaru ML, Donahoe J, Corral T, Reilly KM, Brophy S, Pellicer A, et al: Neurofibromatosis 1 (NF1) heterozygosity results in a cell-autonomous growth advantage for astrocytes. Glia 33: 314-323, 2001

5. Bajenaru ML, Garbow JR, Perry A, Hernandez MR, Gutmann DH: Natural history of neurofibromatosis 1-associated optic nerve glioma in mice. Ann Neurol 57:119-127, 2005

6. Bajenaru ML, Hernandez MR, Perry A, Zhu Y, Parada LF, Garbow JR, et al: Optic nerve glioma in mice requires astrocyte Nf1 gene inactivation and Nfl brain heterozygosity. Cancer Res 63:8573-8577, 2003

7. Balcer LJ, Liu GT, Heller G, Bilaniuk L, Volpe NJ, Galetta SL, et al: Visual loss in children with neurofibromatosis type 1 and optic pathway gliomas: relation to tumor location by magnetic resonance imaging. Am J Ophthalmol 131:442-445, 2001

8. Banerjee D, Hegedus B, Gutmann DH, Garbow JR: Detection and measurement of neurofibromatosis-1 mouse optic glioma in vivo. Neuroimage 35:1434-1437, 2007

9. Blazo MA, Lewis RA, Chintagumpala MM, Frazier M, McCluggage C, Plon SE: Outcomes of systematic screening for optic pathway tumors in children with Neurofibromatosis Type 1. Am J Med Genet A 127:224-229, 2004

10. Borit A, Richardson EP Jr: The biological and clinical behavior of pilocytic astrocytomas of the optic pathways. Brain 105: 161-187, 1982

11. Brannan CI, Perkins AS, Vogel KS, Ratner N, Nordlund ML, Reid SW, et al: Targeted disruption of the neurofibromatosis type-1 gene leads to developmental abnormalities in heart and various neural crest-derived tissues. Genes Dev 8:1019-1029, 1994

12. Cappelli C, Grill J, Raquin M, Pierre-Kahn A, Lellouch-Tubiana A, Terrier-Lacombe MJ, et al: Long-term follow up of 69 patients treated for optic pathway tumors before the chemotherapy era. Arch Dis Child 79:334-338, 1998

13. Cawthon RM, Weiss R, Xu GF, Viskochil D, Culver M, Stevens $\mathrm{J}$, et al: A major segment of the neurofibromatosis type 1 gene: cDNA sequence, genomic structure, and point mutations. Cell 62:193-201, 1990

14. Chan MY, Foong AP, Heisey DM, Harkness W, Hayward R, Michalski A: Potential prognostic factors of relapse-free survival in childhood optic pathway glioma: a multivariate analysis. Pediatr Neurosurg 29:23-28, 1998 
15. Chateil JF, Soussotte C, Pedespan JM, Brun M, Le Manh C, Diard F: MRI and clinical differences between optic pathway tumors in children with and without neurofibromatosis. Br J Radiol 74: 24-31, 2001

16. Chen SI, Chandna A, Norcia AM, Pettet M, Stone D: The repeatability of best corrected acuity in normal and amblyopic children 4 to 12 years of age. Invest Ophthalmol Vis Sci 47:614-619, 2006

17. Cnossen MH, Stam EN, Cooiman LC, Simonsz HJ, Stroink H, Oranje AP, et al: Endocrinologic disorders and optic pathway gliomas in children with neurofibromatosis type 1. Pediatrics 100:667-670, 1997

18. Combs SE, Schulz-Ertner D, Moschos D, Thilmann C, Huber PE, Debus J: Fractionated stereotactic radiotherapy of optic pathway gliomas: tolerance and long-term outcome. Int J Radiat Oncol Biol Phys 62:814-819, 2005

19. Cummings TJ, Provenzale JM, Hunter SB, Friedman AH, Klintworth GK, Bigner SH, et al: Gliomas of the optic nerve: histological, immunohistochemical (MIB-1 and p53), and MRI analysis. Acta Neuropathol (Berl) 99:563-570, 2000

20. Czyzyk E, Józwiak S, Roszkowski M, Schwartz RA: Optic pathway gliomas in children with and without neurofibromatosis 1 . J Child Neurol 18:471-478, 2003

21. Danoff BF, Kramer S, Thompson N: The radiotherapeutic management of optic nerve gliomas in children. Int J Radiat Oncol Biol Phys 6:45-50, 1980

22. Deliganis AV, Geyer JR, Berger MS: Prognostic significance of type 1 neurofibromatosis (von Recklinghausen Disease) in childhood optic glioma. Neurosurgery 38:1114-1119, 1996

23. Destro M, D’Amico DJ, Gragoudas ES, Brockhurst RJ, Pinnolis MK, Albert DM, et al: Retinal manifestations of neurofibromatosis. Diagnosis and management. Arch Ophthalmol 109: 662-666, 1991

24. Duerr EM, Rollbrocker B, Hayashi Y, Peters N, Meyer-Puttlitz B, Louis DN, et al: PTEN mutations in gliomas and glioneuronal tumors. Oncogene 16:2259-2264, 1998

25. Dutton JJ: Gliomas of the anterior visual pathway. Surv Ophthalmol 38:427-452, 1994

26. Edmonds BT, Wyckoff J, Yeung YG, Wang Y, Stanley ER, Jones $\mathrm{J}$, et al: Elongation factor-1 alpha is an overexpressed actin binding protein in metastatic rat mammary adenocarcinoma. J Cell Sci 109:2705-2714, 1996

27. Erbay SH, Oljeski SA, Bhadelia R: Rapid development of optic glioma in a patient with hybrid phakomatosis: neurofibromatosis type 1 and tuberous sclerosis. AJNR Am J Neuroradiol 25: 36-38, 2004

28. Figarella-Branger D, Daniel L, Andre P, Guia S, Renaud W, Monti G, et al: The PEN5 epitope identifies an oligodendrocyte precursor cell population and pilocytic astrocytomas. Am J Pathol 155:1261-1269, 1999

29. Getz LM, Dobson V, Luna B, Mash C: Interobserver reliability of the Teller Acuity Card procedure in pediatric patients. Invest Ophthalmol Vis Sci 37:180-187, 1996

30. Gnekow AK, Kortmann RD, Pietsch T, Emser A: Low grade chiasmatic-hypothalamic glioma-carboplatin and vincristin chemotherapy effectively defers radiotherapy within a comprehensive treatment strategy-report from the multicenter treatment study for children and adolescents with a low grade gliomaHIT-LGG 1996 - of the Society of Pediatric Oncology and Hematology (GPOH). Klin Padiatr 216:331-342, 2004

31. Gottschalk S, Tavakolian R, Buske A, Tinschert S, Lehmann R: Spontaneous remission of chiasmatic/hypothalamic masses in neurofibromatosis type 1: report of two cases. Neuroradiology 41:199-201, 1999

32. Grill J, Couanet D, Cappelli C, Habrand JL, Rodriguez D, SainteRose C, et al: Radiation-induced cerebral vasculopathy in children with neurofibromatosis and optic pathway glioma. Ann Neurol 45:393-396, 1999

33. Grill J, Laithier V, Rodriguez D, Raquin MA, Pierre-Kahn A,
Kalifa C: When do children with optic pathway tumors need treatment? An oncological perspective in 106 patients treated in a single centre. Eur J Pediatr 159:692-696, 2000

34. Gururangan S, Fisher MJ, Allen JC, Herndon JE II, Quinn JA, Reardon DA, et al: Temozolomide in children with progressive low-grade glioma. Neuro Oncol 9:161-168, 2007

35. Gutmann DH, Giovannini M: Mouse models of neurofibromatosis 1 and 2. Neoplasia 4:279-90, 2002

36. Gutmann DH, Hedrick NM, Li J, Nagarajan R, Perry A, Watson MA: Comparative gene expression profile analysis of neurofibromatosis 1-associated and sporadic pilocytic astrocytomas. Cancer Res 62:2085-2091, 2002

37. Gutmann DH, Loehr A, Zhang Y, Kim J, Henkemeyer M, Cashen A: Haploinsufficiency for the neurofibromatosis 1 (NF1) tumor suppressor results in increased astrocyte proliferation. Oncogene 18:4450-4459, 1999

38. Habiby R, Silverman B, Listernick R, Charrow J: Precocious puberty in children with neurofibromatosis type 1. J Pediatr 126:364-367, 1995

39. Hoffman HJ, Humphreys RP, Drake JM, Rutka JT, Becker LE, Jenkin D, et al: Optic pathway/hypothalamic gliomas: a dilemma in management. Pediatr Neurosurg 19:186-195, 1993

40. Huson S, Jones D, Beck L: Ophthalmic manifestations of neurofibromatosis. Br J Ophthalmol 71:235-238, 1987

41. Jacks T, Shih TS, Schmitt EM, Bronson RT, Bernards A, Weinberg RA: Tumour predisposition in mice heterozygous for a targeted mutation in Nf1. Nat Genet 7:353-361, 1994

42. Jahraus CD, Tarbell NJ: Optic pathway gliomas. Pediatr Blood Cancer 46:586-596, 2006

43. Kernan JC, Horgan MA, Piatt JH, D'Agostino A: Spontaneous involution of a diencephalic astrocytoma. Pediatr Neurosurg 29: 149-153, 1998

44. King A, Listernick R, Charrow J, Piersall L, Gutmann DH: Optic pathway gliomas in neurofibromatosis type 1: the effect of presenting symptoms on outcome. Am J Med Genet A 122:95-99, 2003

45. Kornreich L, Blaser S, Schwarz M, Shuper A, Vishne TH, Cohen IJ, et al: Optic pathway glioma: correlation of imaging findings with the presence of neurofibromatosis. AJNR Am J Neuroradiol 22:1963-1969, 2001

46. Kuenzle C, Weissert M, Roulet E, Bode H, Schefer S, Huisman T, et al: Follow-up of optic pathway gliomas in children with neurofibromatosis type 1. Neuropediatrics 25:295-300, 1994

47. Kuo DJ, Weiner HL, Wisoff J, Miller DC, Knopp EA, Finlay JL: Temozolomide is active in childhood, progressive, unresectable, low-grade gliomas. J Pediatr Hematol Oncol 25:372-378, 2003

48. Lacaze E, Kieffer V, Streri A, Lorenzi C, Gentaz E, Habrand JL, et al: Neuropsychological outcome in children with optic pathway tumors when first-line treatment is chemotherapy. Br J Cancer 89:2038-2044, 2003

49. Lau N, Feldkamp MM, Roncari L, Loehr AH, Shannon P, Gutmann DH, et al: Loss of neurofibromin is associated with activation of RAS/MAPK and PI3-k/AKT signaling in a neurofibromatosis 1 astrocytoma. J Neuropathol Exp Neurol 59:759-767, 2000

50. Lee AG, Dutton JJ: A practice pathway for the management of gliomas of the anterior visual pathway: an update and an evidence-based approach. Neuro-ophthalmology 22:139-155, 1999

51. Lewis RA, Gerson LP, Axelson KA, Riccardi VM, Whitford RP: von Recklinghausen neurofibromatosis. II. Incidence of optic gliomata. Ophthalmology 91:929-935, 1984

52. Listernick R, Charrow J, Greenwald M, Mets M: Natural history of optic pathway tumors in children with neurofibromatosis type 1: a longitudinal study. J Pediatr 125:63-66, 1994

53. Listernick R, Charrow J, Gutmann DH: Comments on neurofibromatosis 1 and optic pathway tumors. Am J Med Genet 102:105, 2001

54. Listernick R, Charrow J, Tomita T, Goldman S: Carboplatin ther- 
apy for optic pathway tumors in children with neurofibromatosis type-1. J Neurooncol 45:185-190, 1999

55. Listernick R, Darling C, Greenwald M, Strauss L, Charrow J: Optic pathway tumors in children: the effect of neurofibromatosis type 1 on clinical manifestations and natural history. J Pediatr 127:718-722, 1995

56. Listernick R, Ferner RE, Liu GT, Gutmann DH: Optic pathway gliomas in neurofibromatosis-1: controversies and recommendations. Ann Neurol 61:189-198, 2007

57. Listernick R, Ferner RE, Piersall L, Sharif S, Gutmann DH, Charrow J: Late-onset optic pathway tumors in children with neurofibromatosis 1. Neurology 63:1944-1946, 2004

58. Listernick R, Louis DN, Packer RJ, Gutmann DH: Optic pathway gliomas in children with neurofibromatosis 1: consensus statement from the NF1 Optic Pathway Glioma Task Force. Ann Neurol 41:143-149, 1997

59. Liu GT, Brodsky MC, Phillips PC, Belasco J, Janss A, Golden JC, et al: Optic radiation involvement in optic pathway gliomas in neurofibromatosis. Am J Ophthalmol 137:407-414, 2004

60. Luh GY, Bird CR: Imaging of brain tumors in the pediatric population. Neuroimaging Clin N Am 9:691-716, 1999

61. Lund AM, Skovby F: Optic gliomas in children with neurofibromatosis type 1. Eur J Pediatr 150:835-838, 1991

62. Mahgoub N, Taylor BR, Le Beau MM, Gratiot M, Carlson KM, Atwater SK, et al: Myeloid malignancies induced by alkylating agents in Nf1 mice. Blood 93:3617-3623, 1999

63. Mahoney DH Jr, Cohen ME, Friedman HS, Kepner JL, Gemer L, Langston JW, et al: Carboplatin is effective therapy for young children with progressive optic pathway tumors: a Pediatric Oncology Group phase II study. Neuro Oncol 2:213-220, 2000

64. Nishio S, Takeshita I, Fukui M, Yamashita M, Tateishi J: Anaplastic evolution of childhood optico-hypothalamic pilocytic astrocytoma: report of an autopsy case. Clin Neuropathol 7: 254-258, 1988

65. O'Connell P, Cawthon R, Xu GF, Li Y, Viskochil D, White R: The neurofibromatosis type 1 (NF1) gene: identification and partial characterization of a putative tumor suppressor gene. J Dermatol 19:881-884, 1992

66. Packer RJ, Ater J, Allen J, Phillips P, Geyer R, Nicholson HS, et al: Carboplatin and vincristine chemotherapy for children with newly diagnosed progressive low-grade gliomas. J Neurosurg 86:747-754, 1997

67. Packer RJ, Bilaniuk LT, Cohen BH, Braffman BH, Obringer AC, Zimmerman RA, et al: Intracranial visual pathway gliomas in children with neurofibromatosis. Neurofibromatosis 1:212-222, 1988

68. Parazzini C, Triulzi F, Bianchini E, Agnetti V, Conti M, Zanolini $\mathrm{C}$, et al: Spontaneous involution of optic pathway lesions in neurofibromatosis type 1: serial contrast MR evaluation. AJNR Am J Neuroradiol 16:1711-1718, 1995

69. Parsa CF, Hoyt CS, Lesser RL, Weinstein JM, Strother CM, MuciMendoza R, et al: Spontaneous regression of optic gliomas: thirteen cases documented by serial neuroimaging. Arch Ophthalmol 119:516-529, 2001

70. Pepin SM, Lessell S: Anterior visual pathway gliomas: the last 30 years. Semin Ophthalmol 21:117-124, 2006

71. Phillips SM, Bendall AJ, Ramshaw IA: Isolation of gene associated with high metastatic potential in rat mammary adenocarcinomas. J Natl Cancer Inst 82:199-203, 1990

72. Piccirilli M, Lenzi J, Delfinis C, Trasimeni G, Salvati M, Raco A: Spontaneous regression of optic pathways gliomas in three patients with neurofibromatosis type I and critical review of the literature. Childs Nerv Syst 22:1332-1337, 2006

73. Pinna A, Demontis S, Maltese G, Dore S, Carta F: Absence of the greater sphenoid wing in neurofibromatosis 1 . Arch Ophthalmol 123:1454, 2005

74. Rubinstein LJ: Pathological features of optic nerve and chiasmatic gliomas. Neurofibromatosis 1:152-158, 1988
75. Rubtsova IV, Parsa CF, Hoyt WF: [Spontaneous regression of familial glioma of the optic nerve in a boy with suspected neurofibromatosis 1 (Recklinghausen's disease).] Vestn Oftalmol 114:48-51, 1998

76. Sharif S, Ferner R, Birch JM, Gillespie JE, Gattamaneni HR, Baser ME, et al: Second primary tumors in neurofibromatosis 1 patients treated for optic glioma: substantial risks after radiotherapy. J Clin Oncol 24:2570-2575, 2006

77. Sherman LS, Atit R, Rosenbaum T, Cox AD, Ratner N: Single cell Ras-GTP analysis reveals altered Ras activity in a subpopulation of neurofibroma Schwann cells but not fibroblasts. J Biol Chem 275:30740-30745, 2000

78. Sheta SS, el-Toukhy EA, Khattab HM: Optic nerve glioma and colonic polyposis: report of a new association. Acta Paediatr 93:1259-1260, 2004

79. Singhal S, Birch JM, Kerr B, Lashford L, Evans DG: Neurofibromatosis type 1 and sporadic optic gliomas. Arch Dis Child 87:65-70, 2002

80. Stiebel-Kalish H, Lusky M, Yassur Y, Kalish Y, Shuper A, Erlich $\mathrm{R}$, et al: Swedish interactive thresholding algorithm fast for following visual fields in prepubertal idiopathic intracranial hypertension. Ophthalmology 111:1673-1675, 2004

81. Tada K, Kochi M, Saya H, Kuratsu J, Shiraishi S, Kamiryo T, et al: Preliminary observations on genetic alterations in pilocytic astrocytomas associated with neurofibromatosis 1 . Neuro Oncol 5:228-234, 2003

82. Takenchi H, Kabuto M, Sato K, Kubota T: Chiasmal gliomas with spontaneous regression: proliferation and apoptosis. Childs Nerv Syst 13:229-233, 1997

83. Thiagalingam S, Flaherty M, Billson F, North K: Neurofibromatosis type 1 and optic pathway gliomas: follow-up of 54 patients. Ophthalmology 111:568-577, 2004

84. Tow SL, Chandela S, Miller NR, Avellino AM: Long-term outcome in children with gliomas of the anterior visual pathway. Pediatr Neurol 28:262-270, 2003

85. Ullrich NJ, Robertson R, Kinnamon DD, Scott RM, Kieran MW, Turner CD, et al: Moyamoya following cranial irradiation for primary brain tumors in children. Neurology 68:932-938, 2007

86. Venes JL, Latack J, Kandt RS: Postoperative regression of opticochiasmatic astrocytoma: a case for expectant therapy. Neurosurgery 15:421-423, 1984

87. Verhoeff FH: Tumors of the Optic Nerve. Cytology and Cellular Pathology of the Nervous System. New York: Hafner, 1965, pp 1029-1039

88. Wilson WB, Feinsod M, Hoyt WF, Nielsen SL: Malignant evolution of childhood chiasmal pilocytic astrocytoma. Neurology 26: 322-325, 1976

89. Wolsey DH, Larson SA, Creel D, Hoffman R: Can screening for optic nerve gliomas in patients with neurofibromatosis type I be performed with visual-evoked potential testing? J Aapos 10:307-311, 2006

90. Wong JY, Uhl V, Wara WM, Sheline GE: Optic gliomas. A reanalysis of the University of California, San Francisco experience. Cancer 60: 1847-1855, 1987

91. Xu GF, O’Connell P, Viskochil D, Cawthon R, Robertson M, Culver $\mathrm{M}$, et al: The neurofibromatosis type 1 gene encodes a protein related to GAP. Cell 62:599-608, 1990

92. Zhu Y, Harada T, Liu L, Lush ME, Guignard F, Harada C, et al: Inactivation of NF1 in CNS causes increased glial progenitor proliferation and optic glioma formation. Development 132: 5577-5588, 2005

Manuscript submitted July 27, 2007.

Accepted September 5, 2007.

Address correspondence to: Brien G. Benoit, M.D., M.Sc, F.R.C.S.C., Division of Neurosurgery, The Ottawa Hospital, Civic Campus, C2, 1053 Carling Avenue, Ottawa, Ontario K1Y 4E9. email: bbenoit@ottawahospital.on.ca. 